\title{
Neutrophil content predicts lymphocyte depletion and anti-PD1 treatment failure in NSCLC
}

Julia Kargl, ${ }^{1,2}$ Xiaodong Zhu, ${ }^{1}$ Huajia Zhang, ${ }^{1}$ Grace H. Y. Yang, ${ }^{1}$ Travis J. Friesen, ${ }^{1}$ Melissa Shipley, ${ }^{1}$ Dean Y. Maeda, ${ }^{3}$ John A. Zebala, ${ }^{3}$ Jill McKay-Fleisch, ${ }^{4}$ Gavin Meredith, ${ }^{4}$ Afshin Mashadi-Hossein, ${ }^{4}$ Christina Baik, ${ }^{1}$ Robert H. Pierce, ${ }^{1}$ Mary W. Redman, ${ }^{1}$ Jeffrey C. Thompson, ${ }^{5}$ Steven M. Albelda, ${ }^{5}$ Hamid Bolouri, ${ }^{6,7}$ and A. McGarry Houghton ${ }^{1,6,8}$

${ }^{1}$ Fred Hutchinson Clinical Research Division, Seattle, Washington, USA. ${ }^{2}$ Otto Loewi Research Center, Division of Pharmacology, Medical University of Graz, Graz, Austria. ${ }^{3}$ Syntrix Pharmaceuticals, Auburn, Washington, USA. ${ }^{4}$ Nanostring Inc., Seattle, Washington, USA. ${ }^{5}$ Division of Pulmonary, Allergy, and Critical Care Medicine, Thoracic Oncology Group, Department of Medicine, University of Pennsylvania Perelman School of Medicine, Philadelphia, Pennsylvania, USA. ${ }^{6}$ Human Biology Division, Fred Hutchinson Cancer Research Division, Seattle, Washington, USA. ${ }^{7}$ Allen Institute for Immunology, Seattle, Washington, USA. ${ }^{8}$ Pulmonary and Critical Care Division, University of Washington, Seattle, Washington, USA.

Immune checkpoint inhibitor (ICI) treatment has recently become a first-line therapy for many nonsmall cell lung cancer (NSCLC) patients. Unfortunately, most NSCLC patients are refractory to ICI monotherapy, and initial attempts to address this issue with secondary therapeutics have proven unsuccessful. To identify entities precluding $C D 8^{+} \mathrm{T}$ cell accumulation in this process, we performed unbiased analyses on flow cytometry, gene expression, and multiplexed immunohistochemical data from a NSCLC patient cohort. The results revealed the presence of a myeloid-rich subgroup, which was devoid of $\mathrm{CD}^{+}$and $\mathrm{CD8}{ }^{+} \mathrm{T}$ cells. Of all myeloid cell types assessed, neutrophils were the most highly associated with the myeloid phenotype. Additionally, the ratio of CD8 ${ }^{+} \mathrm{T}$ cells to neutrophils (CD8/PMN) within the tumor mass optimally distinguished between active and myeloid cases. This ratio was also capable of showing the separation of patients responsive to ICI therapy from those with stable or progressive disease in 2 independent cohorts. Tumor-bearing mice treated with a combination of anti-PD1 and SX-682 (CXCR1/2 inhibitor) displayed relocation of lymphocytes from the tumor periphery into a malignant tumor, which was associated with induction of IFN- $\gamma-$ responsive genes. These results suggest that neutrophil antagonism may represent a viable secondary therapeutic strategy to enhance ICI treatment outcomes.

Authorship note: JK, XZ, and $\mathrm{HZ}$ contributed equally.

Conflict of interest: JMF, GM, and A. Mashadi-Hossein are paid employees of Nanostring Inc. DYM and JAZ are paid employees of Syntrix Pharmaceuticals.

Copyright: (c) 2019, American Society for Clinical Investigation.

Submitted: June 6, 2019 Accepted: October 31, 2019 Published: December 19, 2019

Reference information: /CI Insight. 2019;4(24):e130850. https://doi.org/10.1172/jici. insight.130850.

\section{Introduction}

The introduction of immune checkpoint inhibitor (ICI) therapy for lung cancer - the leading cause of cancer-related deaths worldwide - has revolutionized the field (1-4). Based upon the results of several recent clinical trials, anti-PD1 antibody therapy has now become a first-line therapy for most non-small cell lung cancer (NSCLC) patients, whether as pembrolizumab monotherapy for PDL1-high tumors (5) or in combination with conventional chemotherapy for the PDL1-low group (6). Unfortunately, the majority of ICI-recipient NSCLC patients are either refractory to the treatment or develop resistance to the therapy, despite a favorable initial response. Despite numerous clinical trials employing secondary therapeutics to address the ICI-refractory group, efficacious strategies have not yet emerged in this regard.

Thus far, 5 major markers of favorable response to ICI treatment have been identified for NSCLC patients: (a) cigarette smoke consumption (7), (b) advanced mutational spectral burden (8), (c) CD8 ${ }^{+} \mathrm{T}$ cell infiltration into malignant tumor (9), (d) the IFN- $\gamma$ signature (10), and (e) PDL1 staining by IHC > 50\% (5). Effectively, these 5 factors represent different aspects of the same process. High levels of cigarette smoke consumption result in lung cancers with advanced mutational spectral burden. In turn, the greater the number of genetic mutations present in a lung cancer, the greater the chance that one of these mutations is seen as foreign by the immune system, thereby eliciting an antigen-driven immune response. The result 
would be infiltration of $\mathrm{CD}^{+} \mathrm{T}$ cells, IFN- $\gamma$ release, and an increase in the expression of IFN- $\gamma-$ responsive genes. This type of an immune response would frequently involve a high level of PDL1 expression within the tumor microenvironment (TME) and induction of inhibitory receptor expression (e.g., PD1) by lymphocytes, thus setting the stage for a favorable response to PD1 antibody therapy.

Whereas these factors describe the $\sim 20 \%$ of NSCLC patients responding to ICI monotherapy, the factors mediating ICI-refractory lung cancers - the other $80 \%$ of NSCLC patients treated with anti-PD1 monotherapy - remain poorly understood. Certainly, lung cancers arising in never smokers and light smokers frequently contain relatively few mutations, and these so-called cold tumors rarely elicit antigen-driven immune responses (11). However, this concept does not account for the numerous moderate-to-heavy cigarette smokers who do not benefit from ICI treatment. It is likely that some of these patients fail to benefit from immune-based therapies because their tumors contain a complex TME that either limits lymphocyte proliferation or limits lymphocyte access to malignant portions of tumor (12). Two recent gene expression-based approaches have reported the existence of a "lymphocyte-depleted" phenotype, in which there is ample immune cell content within the tumor but a relatively sparse lymphocyte component $(13,14)$. Although the exact features of such tumors that retard lymphocyte infiltration have not been delineated in any detail, it appears likely that at least a subset of such tumors will harbor myeloid- and/or fibroblast-rich tumor stroma. However, a detailed interrogation of the relative contribution of macrophages versus monocytes versus neutrophils on lymphocyte exclusion has not been performed.

There are 2 key limitations when relying on gene expression data to infer immune cell content and function in solid tumors. First, some myeloid lineage cells are relatively transcriptionally inactive, and their contribution to the TME is therefore underappreciated, if appreciated at all. This is particularly true for neutrophil lineage cells, which represent a significant proportion of immune cells in lung cancers but are rarely part of gene expression signatures defining TME subtypes. Second, the lack of tumor anatomy does not allow for a determination of whether or not tumor-infiltrating lymphocytes have actually infiltrated into malignant tumor, nor an assessment of the TME factors that limit lymphocyte infiltration. To overcome these limitations, we have used a combination of flow cytometry, gene expression studies, and multiplexed-IHC (M-IHC) to identify fundamental immune responses to lung cancer, to determine the key components of lymphocyte-depleted tumors, and to determine their impact on treatment responses to ICI therapy in NSCLC patients.

\section{Results}

Multiparametric flow cytometry identifies fundamental immune response subtypes in NSCLC. To distinguish between favorable and deleterious immune responses in NSCLC, we performed a series of unbiased analyses on $n=68$ cases from a previously reported flow cytometric data set of NSCLC subjects capable of identifying 51 distinct immune populations and subpopulations (15). Initially, we performed a Principal Coordinates Analysis (PCoA) of the flow cytometric data generated from both the lung tumor specimens and from nonadjacent lung tissue ( $>3 \mathrm{~cm}$ removed from the tumor). The results showed that the majority of the lung tumor flow immune profiles mirrored those identified in the lung tissue (Supplemental Figure 1A; supplemental material available online with this article; https://doi.org/10.1172/jci.insight.130850DS1). However, $n=15$ of the lung tumor specimens separated from the remainder of the cohort. This immune "Unique" subgroup was defined by substantial increases in $\mathrm{T}$ cell infiltration within the tumor specimen, most significantly for $\mathrm{CD}^{+}, \mathrm{CD}^{+} \mathrm{TIM}^{+}, \mathrm{CD} 8^{+} \mathrm{IFN}-\gamma^{+}$and $\mathrm{CD} 8^{+} \mathrm{PD} 1^{+} \mathrm{T}$ cells (Supplemental Figure 1B). Notably, the immune Unique subgroup also displayed statistically significant reductions in both neutrophil $\left(\mathrm{CD}_{6} 6 \mathrm{~b}^{+}\right)$and macrophage $\left(\mathrm{CD} 68^{+}\right)$content

We also performed unsupervised hierarchical clustering of the flow data set, which further subdivided the fundamental immune responses in the cohort (Figure 1A). A small number of cases $(n=10)$ displayed very little immune cell content of any kind and were labeled "Inert" tumors, to distinguish them from previously described "Cold" tumors, in which T cell content is sparse, though the remainder immune cell content is not defined. We did not attempt to study these cases further. Three additional major subgroups were identified: a group displaying robust $\mathrm{CD} 8^{+} \mathrm{T}$ cell infiltration, which we deemed the "Active" group; a group with sparse $\mathrm{T}$ cell infiltration but abundant myeloid lineage cell content, which we termed the "Myeloid" group; and a less well-defined group that displayed robust Th17 cytokine family member staining, which was termed immune "Indeterminate." The Active group housed the majority of the immune Unique profiles identified using PCoA, as above. 

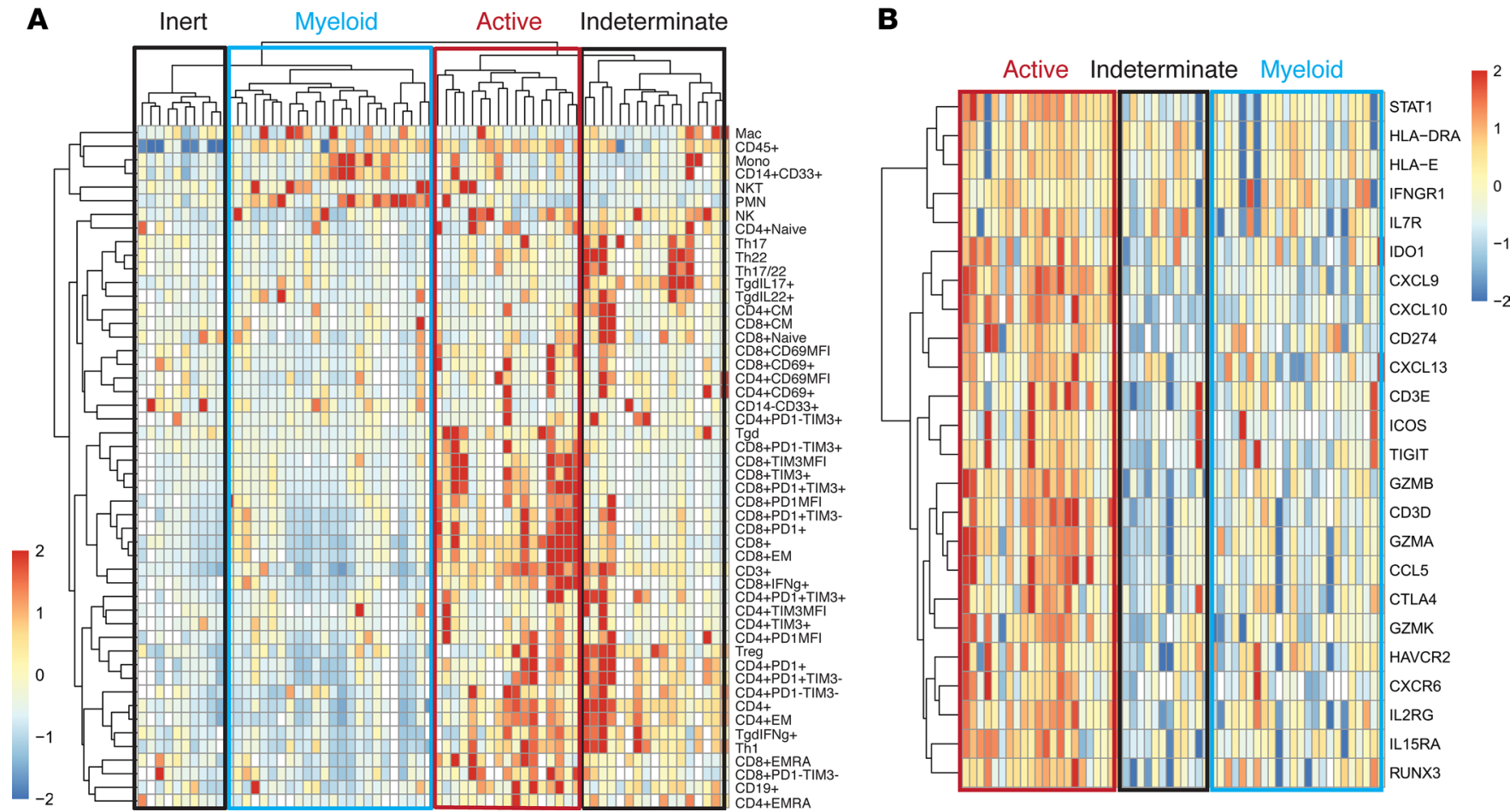

Figure 1. Unsupervised hierarchical clustering identifies immune response subtypes. (A) Unsupervised hierarchical clustering of $n=68$ NSCLC tumor specimens using 51 distinct immune cell populations and subpopulations. Each population is expressed as relative abundance. (B) Heatmap of selected genes from Nanostring Immune Profiling gene expression panel $(n=58)$.

IFN- $\gamma$-induced gene expression separates Active tumors from the other immune subtypes. A simple comparison of the flow profiles of the 4 subgroups revealed that the $\mathrm{CD} 8^{+} \mathrm{T}$ cell population and subpopulations were significantly higher in what we have termed the immune "Active" phenotype, as compared with the other groups (Figure 1A). Analysis of the Nanostring Immune Profiling panel from the entire cohort showed an increase in IFN- $\gamma$ responsive genes in the Active group (Figure 1B). Indeed, the majority of immune Active cases (16 of 22) displayed robust IFN- $\gamma$ gene signatures, but such gene products were rarely encountered in the immune Indeterminate group ( 3 of 13). Notably, none of the Myeloid cases displayed the IFN- $\gamma$ signature.

In attempts to identify the major drivers of the Myeloid group, we specifically compared this group with the immune Active group, which possesses cellular populations and gene expression signatures predictive of favorable outcomes. The genes most highly associated with the immune Active group included well-described mediators of $\mathrm{T}$ cell recruitment and proliferation, such as CXCL9, CXCL10, and IL2 receptor- $\beta(I L 2 R \beta)$. Granzyme B $(G Z M B)$ expression - indicative of cytotoxic T lymphocyte function and STAT1 expression, indicative of $\mathrm{Th} 1\left(\mathrm{CD} 4^{+} \mathrm{IFN}-\gamma^{+}\right)$cellular differentiation (16) - were also highly expressed in this group (Figure 2A). In contrast, most of the genes highly associated with the Myeloid phenotype are involved in neutrophil lineage recruitment and function. Toll-IL-1 receptor (TIR) domain containing adaptor protein (TIRAP) and IL1R2 encode innate and IL-1 $\beta$ signaling proteins that promote inflammatory responses rich in myeloid cells. CXCL5 is a well-defined neutrophil chemokine and lipocalin-2 (LCN2), also known as neutrophil-gelatinase associated lipocalin (NGAL), is an iron siderophore housed in neutrophil granules (17). Some of the neutrophil signature identified in the Myeloid group implicates the myeloid-derived suppressor cell (MDSC) subset of neutrophils (PMN-MDSC), as tumor necrosis factor receptor superfamily member 10B (TNFRSF10B) encodes a death receptor (death receptor-5; DR5), recently shown to be differentially expressed by PMN-MDSC over traditional PMN (18).

The cellular populations driving the Active phenotype were activated $\mathrm{CD} 4^{+}$and $\mathrm{CD} 8^{+} \mathrm{T}$ cells, those that highly express inhibitory receptors such as PD1 (Figure 2B). Both effector memory (CCR7 CD45RA) and effector memory RA (CCR7 $\left.\mathrm{CD} 45 \mathrm{RA}^{+}\right)$cells of $\mathrm{CD}^{+}$and $\mathrm{CD}^{+}$cellular lineages were highly represented in this group, as well. Not surprisingly, $\mathrm{CD} 19^{+} \mathrm{B}$ cells were also significantly more abundant in Active than Myeloid cases. Consistent with the gene expression data above, neutrophils $\left(\mathrm{CD} 6 \mathrm{~b}^{+}\right)$were the cellular 
A
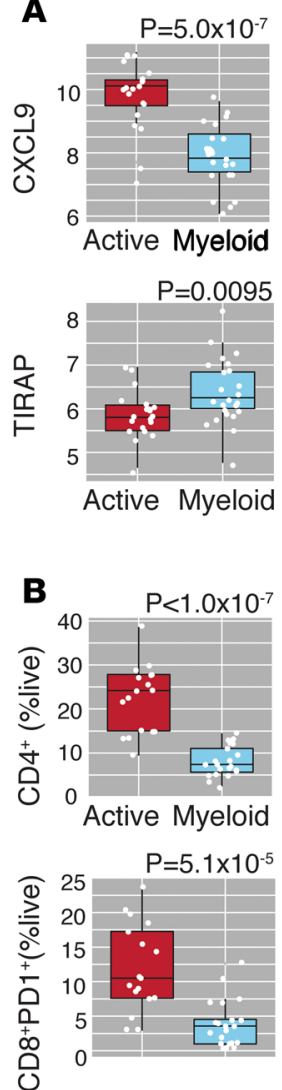
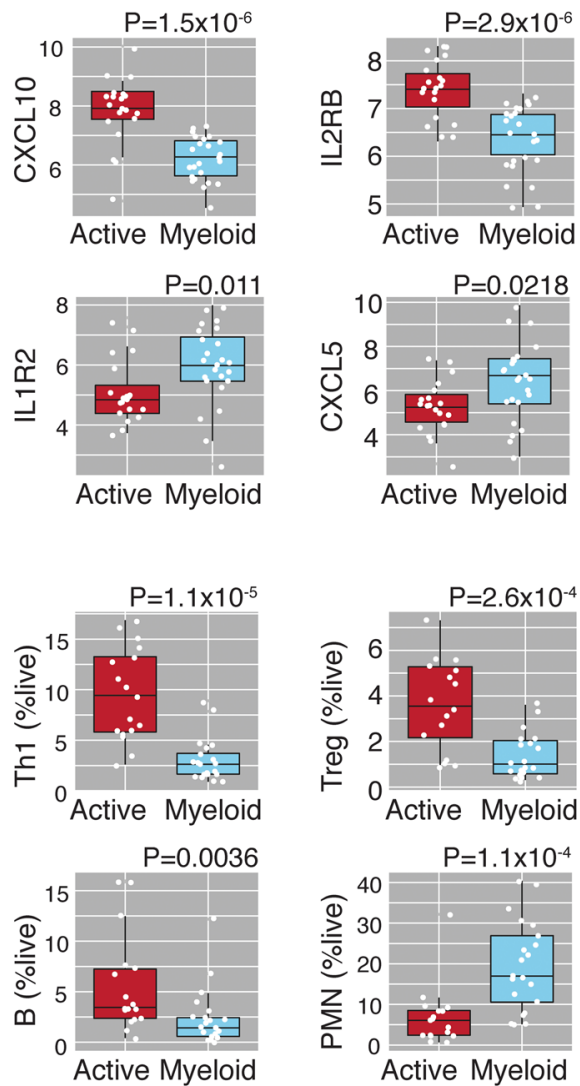

Active Myeloid
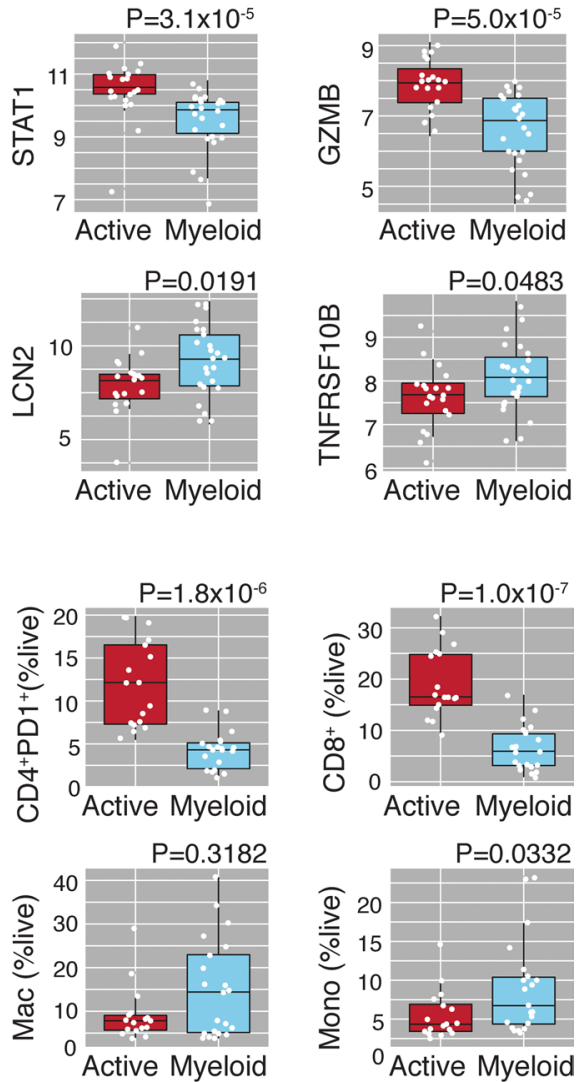

Figure 2. Gene expression and flow cytometric population differences by immune subtype. (A) Comparison of expression for indicated genes between immune Active $(n=22)$ and Myeloid $(n=24)$ tumors. Data provided as normalized relative gene expression from a panel of housekeeping genes. $P$ value as indicated (Student's $t$ test). (B) Comparison of flow cytometric data for indicated populations between immune Active ( $n=22)$ and Myeloid $(n=24)$ tumors. Each population expressed as percent live cells. $P$ value as indicated (Student's $t$ test). PMN, polymorphonuclear leukocyte.

population most highly associated with the Myeloid phenotype $\left(P=1.1 \times 10^{-4}\right)$ (Figure $\left.2 \mathrm{~B}\right)$. Monocytes $\left(\mathrm{CD} 14^{+} \mathrm{CD} 68^{-}\right)$were also significantly higher in Myeloid cases than in Active cases $(P=0.0332)$. Although substantially higher in a small number of Myeloid cases, macrophage content was not significantly higher in the Myeloid group as compared with the Active group $(P=0.3182)$.

Since neutrophil content has previously been reported to be increased in lung squamous cell carcinoma (LUSQ) as compared with lung adenocarcinoma (LUAD) cases, the 2 major histologic subtypes of NSCLC, we examined the possibility that NSCLC histologic subtype could impact the immune phenotype. However, we did not identify any statistically significant differences between the 4 immune subgroups as a function of LUSQ versus LUAD histology by $\chi^{2}$ test (Supplemental Table 1).

We sought to validate the key finding that neutrophils (Myeloid phenotype) and CD8 ${ }^{+} \mathrm{T}$ cells (Active phenotype) were anticorrelated. To accomplish this, we analyzed the LUAD TCGA database. We assessed immune cell normalized gene expression data from 57 cancer-control LUAD TCGA-paired samples (tumor-normal lung pairs; Supplemental Figure 2, A and B). Immune cell subset behavior was found to be comparable with flow cytometric analysis using log-transformed data. Specifically, neutrophil content negatively predicted CD8 ${ }^{+}$cellular content, whereas a more generic myeloid lineage marker (CD14) did not (Supplemental Figure 2, C and D).

M-IHC identifies cellular drivers of the immune subphenotypes. Several studies encompassing multiple tumor types have demonstrated that the ability of $\mathrm{CD}^{+} \mathrm{T}$ cells to infiltrate into the malignant portions of a tumor confers both improved overall survival and favorable response to ICI therapy $(9,19,20)$. Topographically, both LUAD and LUSQ display repetitive areas of tumor-stroma interface and not a simple cancer mass versus adjacent tissue dichotomy. To determine the impact of the intratumor location of $\mathrm{CD}^{+}$cells and the cellular lineages that comprise the Myeloid phenotype, we developed a potentially novel 7-color M-IHC panel on the Vectra-3 platform (Perkin-Elmer) and stained and analyzed all of the cases from the resection cohort, described above. We validated 6 antibodies (CD8, 
CD14, CD68, HLA-DR, CD66b, Cytokeratin) on the panel (Supplemental Tables 2 and 3), allowing for detailed interrogation of the association of myeloid lineage cell types with the cellular content and location of $\mathrm{CD}^{+} \mathrm{T}$ cells $\left(\mathrm{CD}^{+}\right)$(Figure 3A and Supplemental Figure 3). The myeloid lineage cells studied here were macrophages $\left(\mathrm{CD}^{+} 8^{+}\right)$, monocytes $\left(\mathrm{CD} 14^{+} \mathrm{CD} 68^{-} \mathrm{HLA}-\mathrm{DR} \mathrm{R}^{+}\right)$, monocytic-MDSC (M-MDSC; $\left.\mathrm{CD}_{14}{ }^{+} \mathrm{CD} 68^{-} \mathrm{HLA}^{-D R^{-}}\right)$, and neutrophils $\left(\mathrm{CD} 6 \mathrm{~b}^{+}\right)$. These cellular definitions were adopted from a recent consensus statement on MDSC nomenclature (21).

After completion of the staining procedure, multispectral images (MSI)were obtained from each slide to allow for further interrogation using HALO image analysis software (Indica Labs), which resulted in the generation of spatial plots to facilitate cellular analysis (Figure 3B). At least 20 stamps, or region of interest (ROI) fields, were acquired from each slide at $\times 20$. Stamps acquired from each slide encompassed the disparate regions of tumor specimens, including central tumor, tumor-stroma interface, tertiary lymphoid structure or lymphoid aggregates (LA), adjacent lung tissue, and necrotic tumor. Vascular structures (and any cells contained within them) were excluded from the analysis. The cellular content of each image was reported as a function of its tumoral location: malignant tumor, stroma, LA, necrotic tumor, or adjacent lung tissue. Additional details are provided in the Methods and Supplemental Figure 4.

To identify the cell types and locations driving the immune Active and Myeloid phenotypes, we initially performed unsupervised hierarchical clustering of all 1,983 stamps acquired from the resection cohort (Figure 3C). Each of the 5 cell types studied in detail were predominantly located in between 2 and 4 clusters. When directly comparing the immune Active versus Myeloid phenotypes, we observed that CD ${ }^{+}$ $\mathrm{T}$ cells were highly significantly increased in the stroma, tumor, and combined stroma plus tumor compartments of the Active cases (Figure 3, D-F). M-MDSC content was surprisingly increased in the Active tumor and stroma compared with the Myeloid compartments. Macrophage content did not preferentially localize with either phenotype, being significantly higher in the stroma but significantly lower in the tumor compartment, of Myeloid tumors. There was no significant difference in macrophage content in the combined tumor plus stroma analysis. Similarly, monocytes did not associate with the Myeloid phenotype in any of the metrics assessed. Of all cell types analyzed, only neutrophil content was higher in the tumor, stroma, and combined stroma plus tumor analyses, specifically in the Myeloid cases compared with the Active cases (Figure 3, D-F). Since $\mathrm{CD}^{+} \mathrm{T}$ cells were most highly associated with immune Active cases and neutrophils were the immune cell population most highly associated with the Myeloid phenotype, we calculated CD8/CD66b ratios using CD66b content from both tumor and stroma (i.e., comparing the CD8 content in the tumor [CD8T] with the neutrophil content in the stroma [CD66bS]). This metric statistically distinguished immune Active from Myeloid cancers with a high degree of significance, whether using stromal (CD66bS) or tumoral (CD66bT) neutrophil content (Figure 3, G and H).

Tumor CD8/PMN ratios are associated with ICI treatment failure in NSCLC. To test the concept that CD8/ CD66b ratios would predict response to ICI treatment in NSCLC patients, we generated a cohort of anti-PD1/PDL1-recipient NSCLC patients for which pretreatment FFPE tissues specimens were available (Supplemental Table 4). All available imaging studies for each subject were utilized to determine the response evaluation criteria in solid tumors (RECIST) V1.1 categorization, as previously described (22). Each patient was assigned to either the complete response (CR), partial response (PR), stable disease (SD), or progressive disease (PD) category. Since our cohort only possessed $n=1 \mathrm{CR}$, we combined the CR and PR groups for analytical purposes. We also abstracted pertinent data from the clinical record to calculate the progression-free survival (PFS) interval for each patient. We utilized similar M-IHC staining methodology and analysis as was described for the resection cohort, above. However, for this panel, we chose to focus on CD8 and CD66b to validate their performance in the resection cohort (Figure 4, A and B). Additionally, we included a CD4 marker, given the prominent role of $\mathrm{CD} 4^{+} \mathrm{T}$ cell content in defining the immune Active phenotype, as defined by flow cytometry.

Initially, we assessed the paired CD4/CD66b and CD8/CD66b relationships within tumor and stroma as a function of RECIST criteria (Figure 4, C and D). The key difference between $\mathrm{CD}^{+}$and $\mathrm{CD} 8^{+} \mathrm{T}$ cells in this regard was that the CD4/CD66b pairs were statistically different in the CR/PR and SD groups as compared with the $\mathrm{PD}$ group $(P=0.017)$, whereas the $\mathrm{CD} 8 / \mathrm{CD} 66 \mathrm{~b}$ pairs were significantly different in the $\mathrm{CR} / \mathrm{PR}$ group than in either the SD or PD group $(P=0.030)$. To facilitate a more facile assessment of the impact of lymphocyte and neutrophil content on ICI treatment outcomes, we calculated CD8T/CD66bS and $\mathrm{CD} 4 \mathrm{~T} / \mathrm{CD} 66 \mathrm{bS}$ ratios. CD4T/CD66bS ratios failed to statistically separate the outcome groups based on RECIST V1.1 criteria (Figure 4E). However, CD8T/CD66bS ratios were significantly different for both 
A
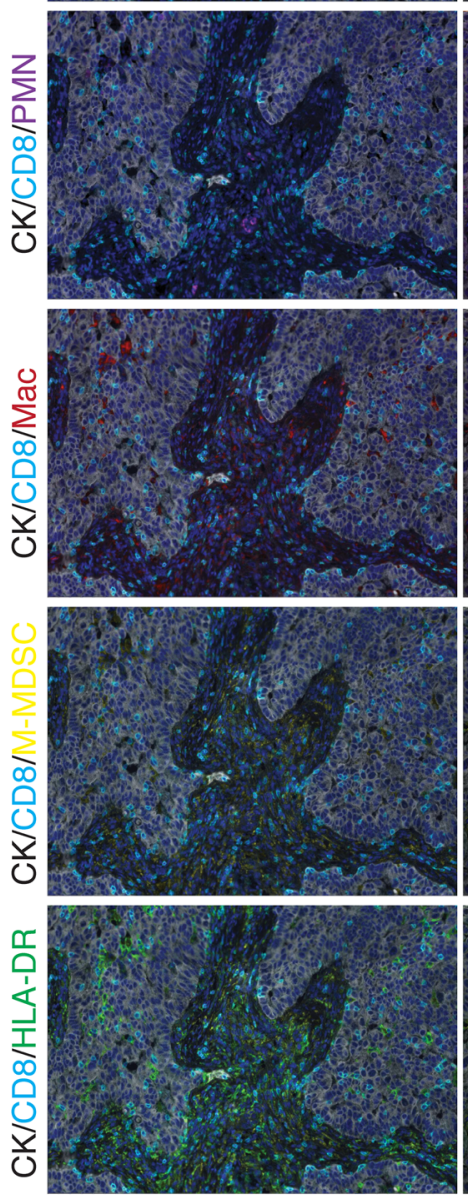

Stroma

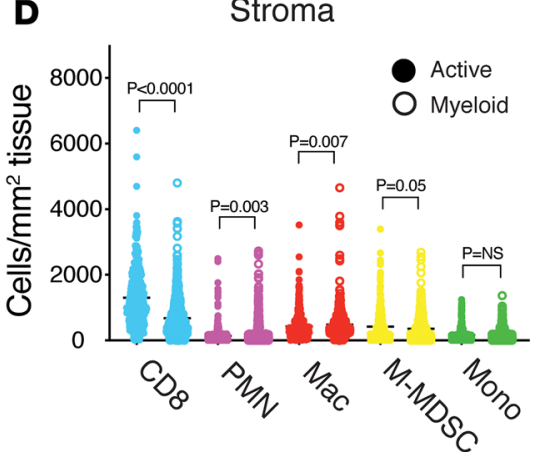

Myeloid
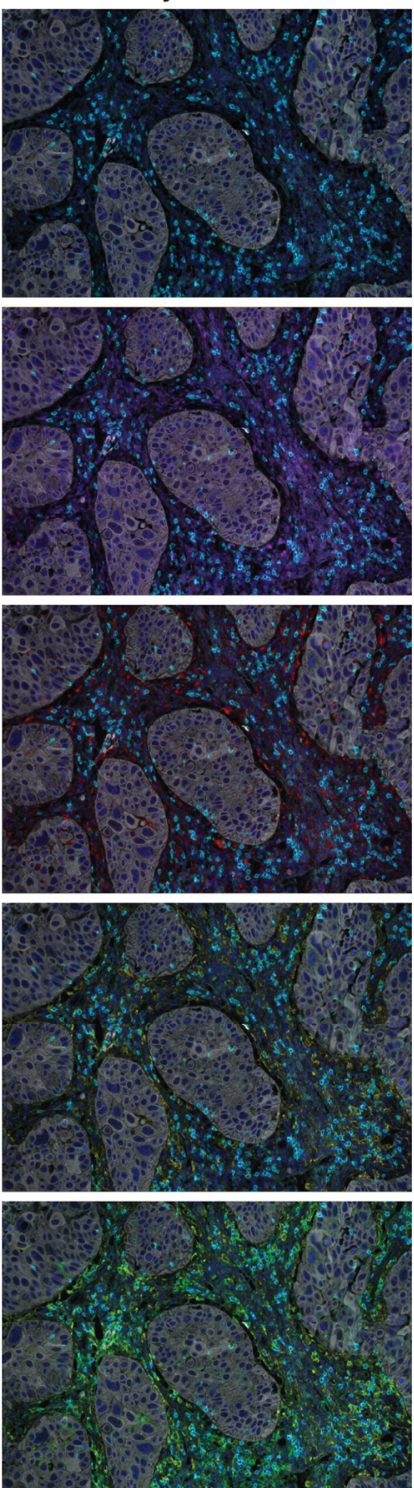

B

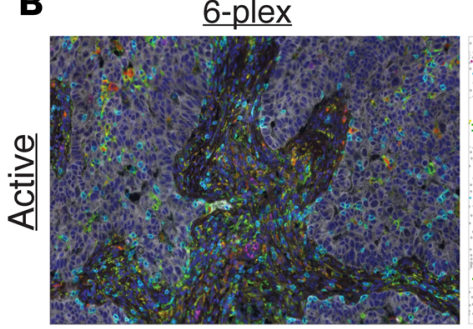

$\square$ CK $\square$ CD8 $\square$ CD14 $\square$ CD66b

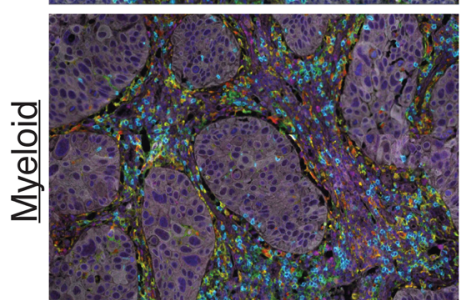

Spatial Plots
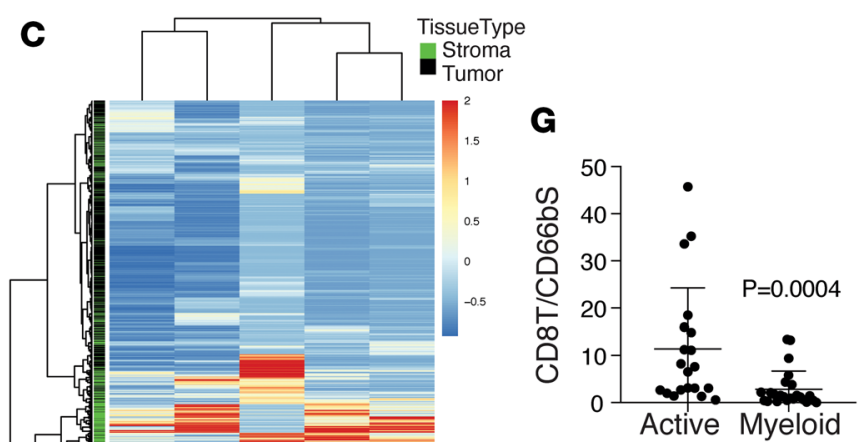

H

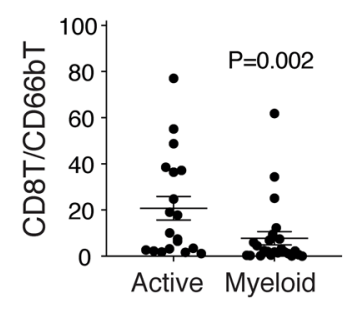

E

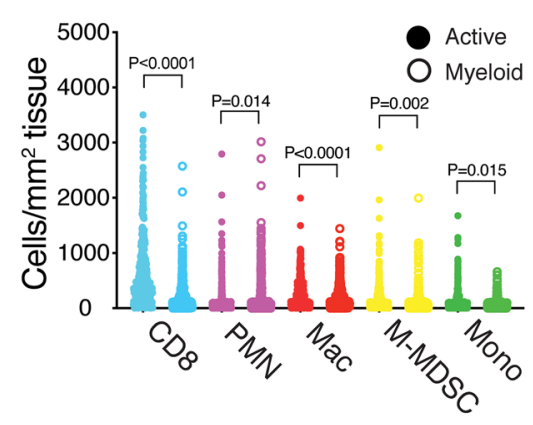

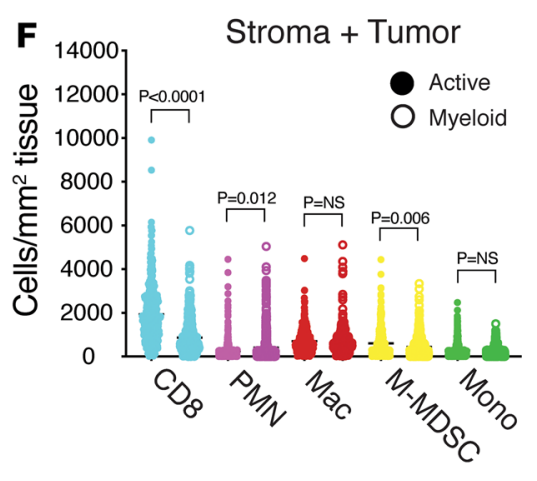

Figure 3. Immune cellular population content by intratumor location. FFPE tumor slides from resection cohort cases $(n=73)$ were stained for CK, CD8, CD66b, CD14, CD68, and HLA-DR, imaged on the Vectra-3 platform and analyzed with HALO. (A) Representative images from immune Active and Myeloid tumors for cytokeratin, CD8, CD66b, CD68, CD14, and HLA-DR, as indicated. Original magnification, $\times 20$. (B) Representative image of a 6-plex and the corresponding HALO spatial plot (threshold data) from an immune Active and Myeloid tumor depicts characteristic immune cell distribution patterns. (C) Clustering analysis of immune cell content as determined by M-IHC analyses. A total of 1,983 fields were analyzed. (D-F) Cellular content from stroma (D), tumor $(\mathbf{E})$, and combined stroma/tumor $(\mathbf{F})$ for indicated populations stratified by tumor immune classification (Active vs. Myeloid tumors). $P$ values as indicated (1-way ANOVA). (G and H) CD8T/CD66bS (G) and CD8T/CD66bT (H) ratios for immune Active $(n=20)$ versus Myeloid ( $n=24)$ tumors. The "T" designates cellular content within malignant tumor, and the " $S$ " designates cellular content within the stroma. $P$ values as indicated (Student's $t$ test). 
A

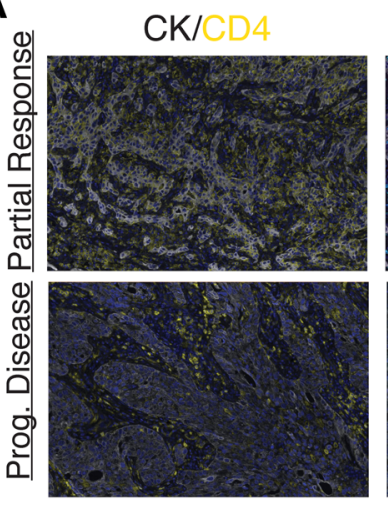

$\mathbf{B}$

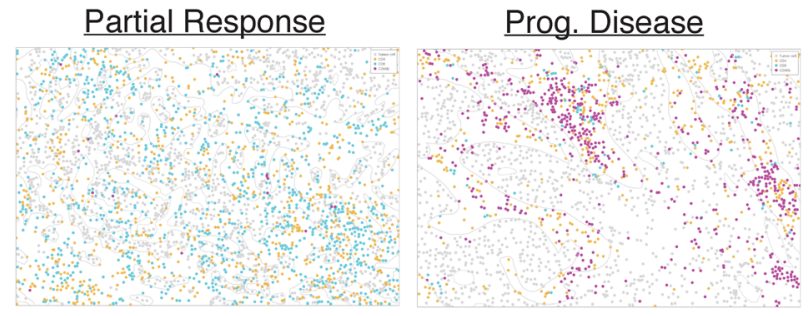

CK/CD8
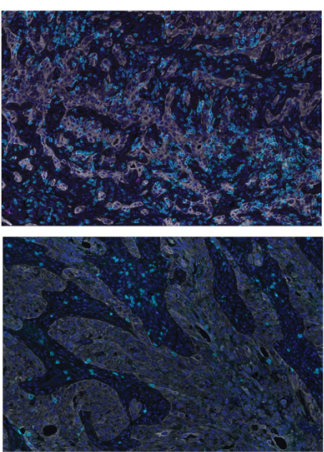

CK/PMN
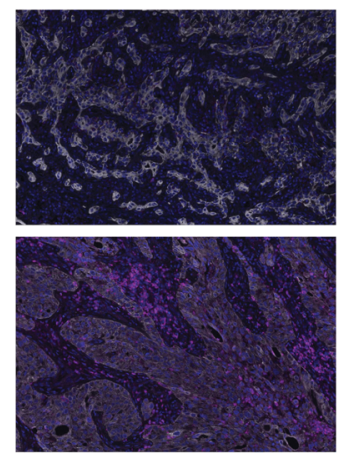

CK/CD8/PMN
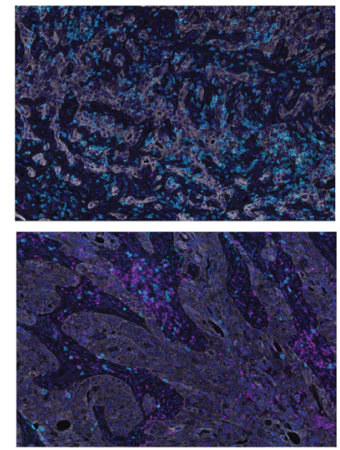

CK/CD8/CD4/PMN
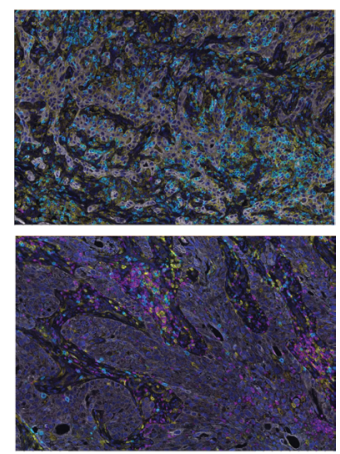

$\mathbf{E}$

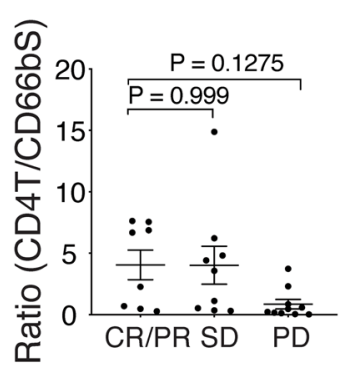

$\mathbf{F}$

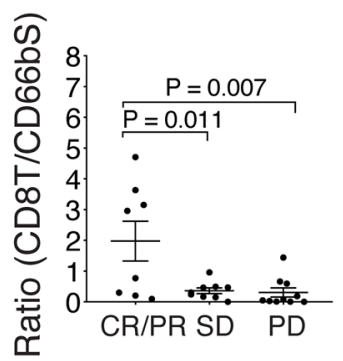

C

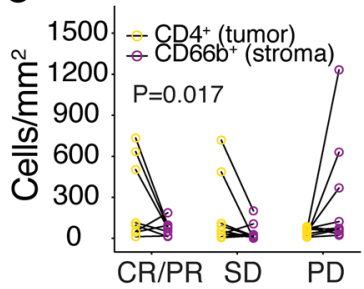

D

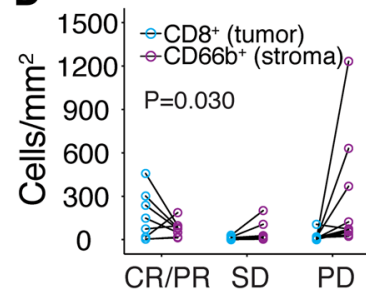

G
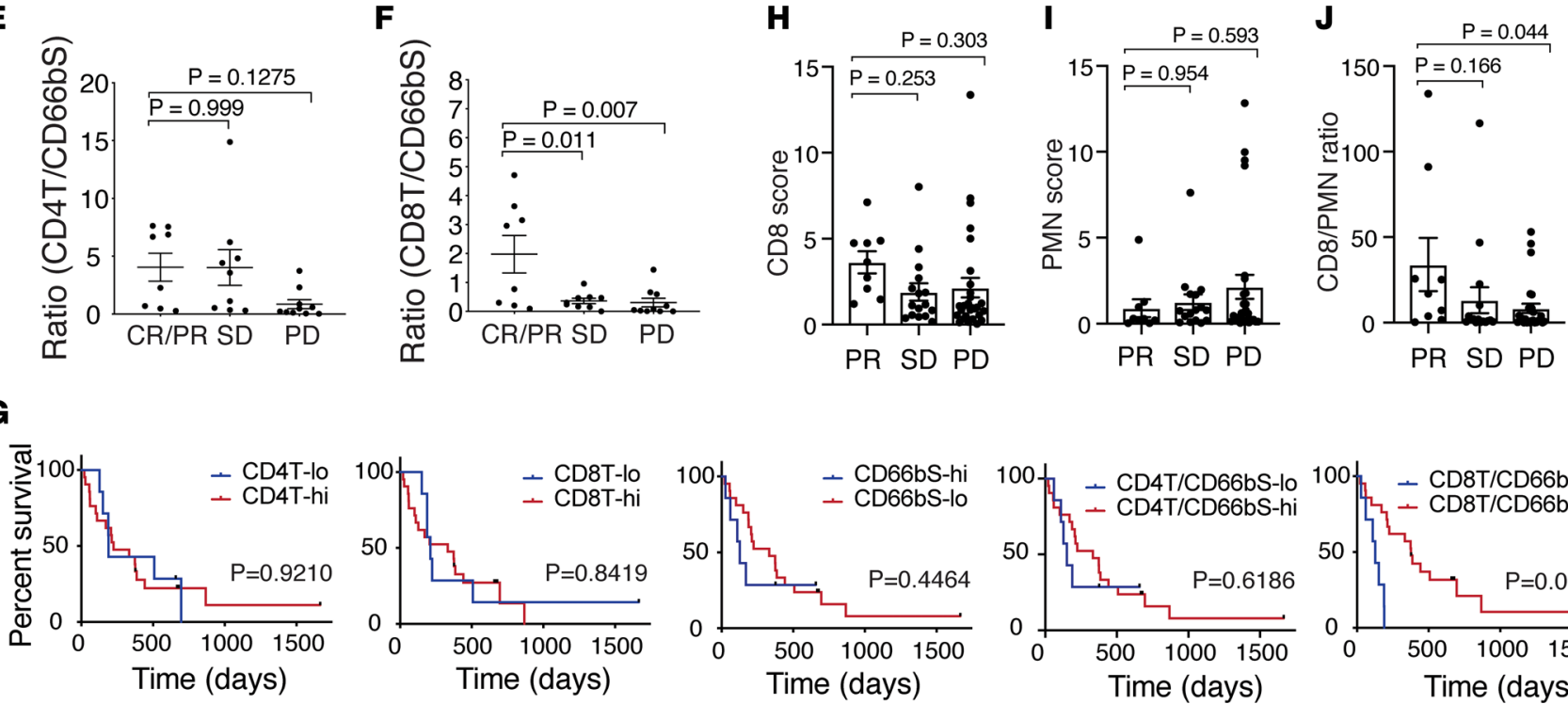

Figure 4. Impact of immune cell populations on ICI treatment outcomes in NSCLC. FFPE tumor slides from a cohort of $n=28$ anti-PD1/PDL1 recipient NSCLC patients (CR/PR $=8, S D=10, P D=10)$ were stained for CK, CD4, CD8, and CD66b, imaged on the Vectra-3 platform, and analyzed with HALO. (A) Representative images from a PR and a PD tumor for cytokeratin, CD4, CD8, CD66b, and the 4-plex panel. Original magnification, $\times 20$. (B) Representative image of a HALO spatial plot from the same cases as in A. (C and D) CD4 content in tumor (CD4T) (C) or CD8 content in tumor (CD8T) paired with CD66b content from stroma (CD66bS) (D) from M-IHC images stratified by response category. $P$ value as indicated (2-way ANOVA). (E and F) CD4T/CD66bS (E) and CD8T/CD66bS (F) ratios from M-IHC images stratified by response category. $P$ values as indicated (1-way ANOVA). (G) Kaplan-Meier life survival curves for the lowest quartiles of CD4T, CD8T, CD4T/CD66bS, and CD8T/CD66bS and the highest quartile of CD66bS vs. the remainder of the cohort. $P$ values as indicated (log-rank test). A validation set consisting of $n=52$ anti-PD1/PDL1 recipient NSCLC patients (CR/PR = 9, SD = 16, PD = 27) was studied using gene expression data to infer immune cell content. (H-J) CD8 score (H), PMN score (I), and CD8/PMN ratio (J) stratified by response category. Data expressed as mean value \pm SEM. $P$ values as indicated (1-way ANOVA).

CR/PR vs. SD $(P=0.011)$ and for $\mathrm{CR} / \mathrm{PR}$ vs. PD $(P=0.007)$ groups (Figure $4 \mathrm{~F})$. In other words, the patients with the highest CD8T/CD66bS ratios displayed the best treatment outcomes. To better understand the factors driving the worst patient outcomes, we calculated the PFS interval for each patient and plotted the survival for the worst quartile of each metric versus the remainder of the cohort (Figure 4G). Somewhat surprisingly, the patients displaying the lowest CD8T content displayed similar PFS intervals 
A

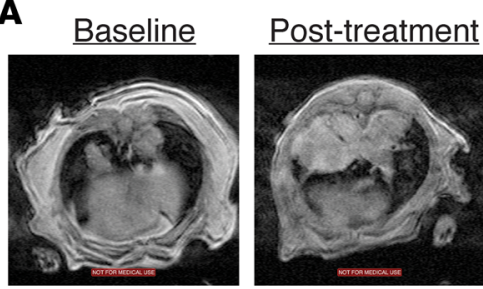

B

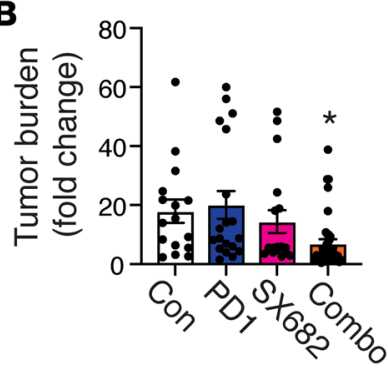

C
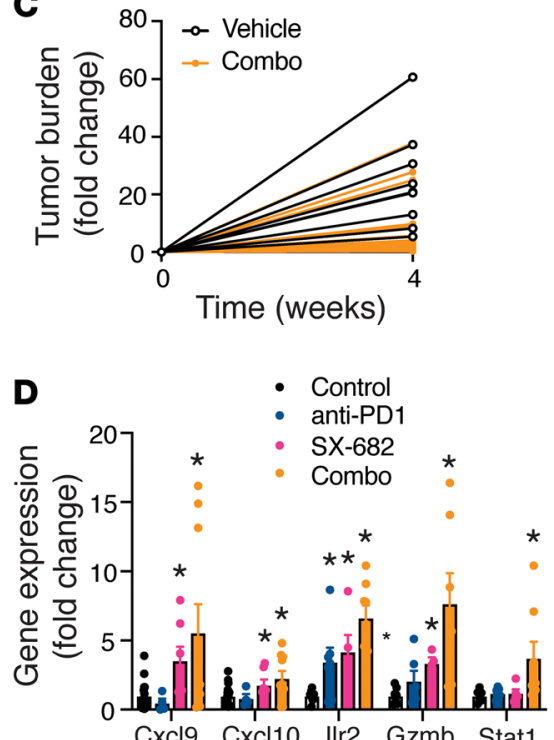

E

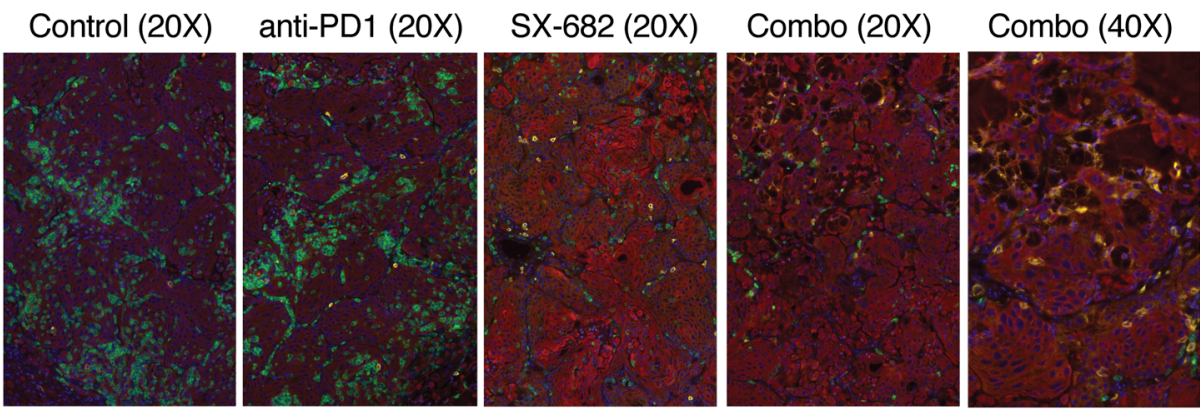

$\mathbf{F}$

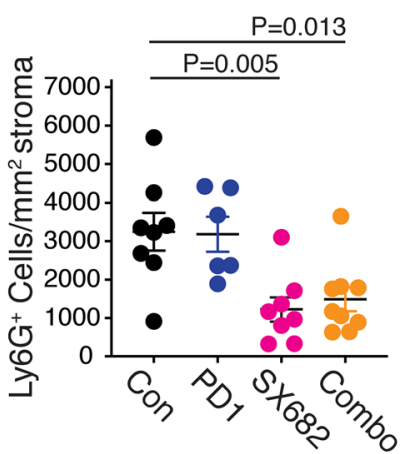

$\mathbf{G}$

H

I

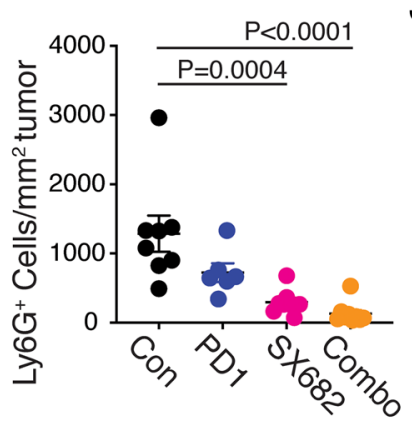

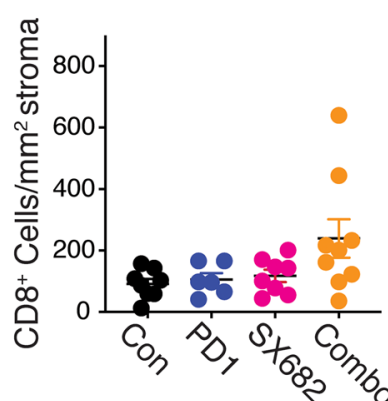

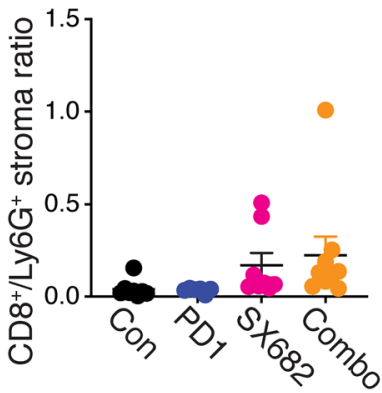

J

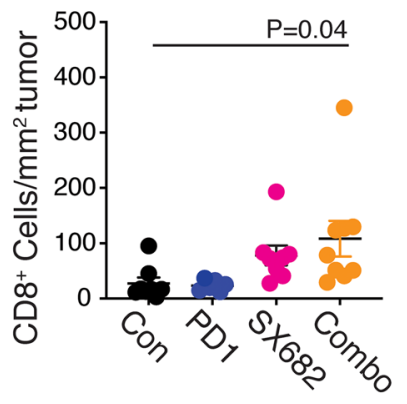

$\mathbf{K}$

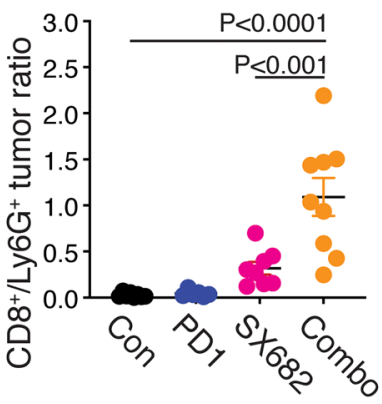

Figure 5. CXCR1/2 antagonism results in increased CD8 ${ }^{+} \mathbf{T}$ cell infiltration into malignant tumor in mice. PL mice were administered $5 \times 10^{7}$ pfu $A d C r e ~ t o$ initiate tumorigenesis, underwent MRI to establish tumor burden, were randomized to a 4-week treatment protocol (control, anti-PD1 antibody, SX-682, or anti-PD1 + SX-682 [combo]), and underwent repeat MRI to establish changes in tumor burden. (A) Representative MRI scans for the control group. (B) Tabulation of tumor burden for each treatment group expressed as the fold change from baseline MRI scan. Control, $n=16 ;$ anti-PD1, $n=19 ; 5 X-682, n=$ 19; combo, $n=38$. ${ }^{*} P<0.05$ (1-way ANOVA). (C) Spider plots for the vehicle control and combo-treated groups from B. (D) qPCR for the indicated genes at the conclusion of the 4-week treatment. Results expressed as fold change in gene expression from control. $n \geq 6$ per group. ${ }^{*} P<0.05$ (1-way ANOVA). (E) Representative M-IHC images for posttreatment tumors. Red, CK (tumor); green, Ly6C (neutrophil); yellow, CD8 (CD8 ${ }^{+} \mathrm{T}$ cell). (F-H) Tabulation of Ly6G ${ }^{+}$ cellular content (F) and CD8 $8^{+}$cellular content (G) expressed as cells $/ \mathrm{mm}^{2}$ tumor stroma tissue, and the ratio of CD8 $8^{+} / \mathrm{Ly}_{6 \mathrm{C}^{+}}$cells $(\mathbf{H})$ within the tumor stroma. (I-K) Tabulation of $\mathrm{Ly}_{6} \mathrm{G}^{+}$cellular content (I) and $\mathrm{CD} 8^{+}$cellular content $(\mathrm{J})$ expressed as cells $/ \mathrm{mm}^{2}$ within malignant tumor tissue, and the ratio of $\mathrm{CD}^{+} / \mathrm{Ly}_{6 \mathrm{G}}+$ cells (K) within malignant tumor. $n \geq 6$ per group. $P$ values as indicated (1-way ANOVA).

as the remainder of the cohort. Similarly, the patients with the lowest CD4T and highest CD66bS content displayed equivalent survival to the remainder of the cohort. The only group displaying decreased PFS were the patients harboring the lowest $\mathrm{CD} 8 \mathrm{~T} / \mathrm{CD} 66 \mathrm{bS}$ ratios $(P=0.0005)$. Thus, the $\mathrm{CD} 8 \mathrm{~T} / \mathrm{CD} 66 \mathrm{bS}$ ratio identified the patients most likely to be nonresponsive to anti-PD1 monotherapy but perhaps most likely to benefit from anti-PD1 therapy when combined with a neutrophil antagonist.

To validate the above findings, we accessed gene expression data (using the HTG EdgeSeq Precision Immuno-Oncology panel) generated on a cohort of $n=52$ NSCLC patients treated with anti-PD1/PDL1 therapy at the University of Pennsylvania. We utilized gene expression sets to infer $\mathrm{CD}^{+} \mathrm{T}$ cell and neutrophil content in these specimens. Neither the CD8 score nor the PMN score statistically predicted treat- 
ment response to anti-PD1/PDL1 by RECIST V1.1 criteria (Figure 4, H and I). However, a ratio of CD8 to PMN scores significantly distinguished between responders (CR/PR) and PD $(P=0.044)$ (Figure $4 \mathrm{~J})$. Thus, the CD8/PMN ratio has proven capable of distinguishing treatment response categories for ICI-recipient NSCLC patients in 2 independent cohorts and utilizing 2 distinct methodologies.

CXCR1/2 inhibition grants access of $C D 8^{+} T$ cells to malignant tumor and restores the IFN- $\gamma$ signature in mice. The fact that tumor CD8/PMN ratios predicted treatment responses to anti-PD1 therapy in human NSCLC suggests that neutrophil antagonism may improve outcomes for these patients, especially for the Myeloid group. To test this concept, we performed a clinical trial in mice utilizing SX-682 (Syntrix Pharmaceuticals), a potentially novel small molecule dual CXCR1/2 inhibitor (23), alone and in combination

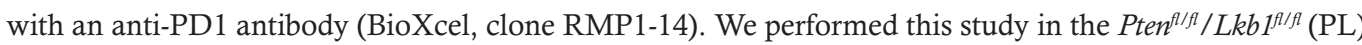
mouse model of lung squamous cell cancer, as this model displays robust neutrophil content (24). PL mice received an intratracheal dose $\left(5.0 \times 10^{7} \mathrm{pfu}\right)$ of adenoviral cre recombinase (AdCre) to delete Pten and Lkb1 and to initiate carcinogenesis. The mice were subjected to MRI 30-weeks after AdCre to identify lung cancer lesions, and this was used to establish the baseline tumor burden. Mice were randomized to receive a 4-week treatment course of either anti-PD1, SX-682, anti-PD1 plus SX-682 (combo), or vehicle control. MRI was repeated after 4 weeks to determine the change in tumor burden (Figure 5A). Tabulation of tumor burden in the different treatment groups revealed that only the combination of anti-PD1 and SX-682 caused a reduction of tumor burden as compared with the vehicle control group (Figure 5, B and C). Neither anti-PD1 nor SX-682 alone significantly reduced tumor burden compared with control.

We utilized quantitative PCR (qPCR) to assess the expression of the 5 genes most highly upregulated in the immune Active group (Figure 2B), which includes IFN- $\gamma$-responsive genes, such as CXCL10. This gene set was increased in SX-682-treated mice, though more so in the combination-treated group (Figure 5D). PD1 antibody monotherapy did not impact the expression of this gene set. Since these data suggest that CXCR1/2 antagonism can restore the IFN- $\gamma$ signature, we crafted an abbreviated M-IHC panel to assess alterations in the tumor location of $\mathrm{CD}^{+} \mathrm{T}$ cells and PMNs induced by CXCR1/2 blockade \pm anti-PD1 antibody treatment (Figure 5E). Similar to the analysis performed in human tissues above, we assessed the impact of these therapies on cellular content within both the stroma and malignant tumor. This analysis showed rather robust neutrophil content within PL tumors that was predominantly located within tumor or putative stroma. $\mathrm{CD}^{+} \mathrm{T}$ cells were sparse in control mice and were typically located within tumor-adjacent lung tissue. $\mathrm{CD}^{+} \mathrm{T}$ cells were not identified infiltrating into malignant tumor in the control group. As expected, the administration of SX-682, alone or in combination with anti-PD1, decreased neutrophil content within both PL stroma and malignant tumor regions (Figure 5, F and I). Although there was trend toward increased $\mathrm{CD}^{+} \mathrm{T}$ cell infiltration into malignant tumor with SX-682 alone, this only reached statistical significance for mice treated with the combination of SX-682 and anti-PD1 (Figure 5J). Notably, $\mathrm{CD}^{+}$cellular content within the stroma was not increased in any of the treatment groups (Figure 5G). Lastly, we calculated stroma and tumor CD8/PMN ratios that proved effective in distinguishing NSCLC patient outcomes in response to anti-PD1 treatment and found that this ratio was significantly increased in the combination-treated group, specifically within the malignant tumor compartment (Figure 5, $\mathrm{H}$ and $\mathrm{K}$ )

\section{Discussion}

Several recent studies have reported unbiased analyses of gene expression data in attempts to identify fundamental immune response subtypes to cancer (12-14). The results of such studies have illustrated the gene expression profiles and inferred immune cell composition distinguishing favorable from unfavorable immune responses to cancer. Most of these endeavors have identified a lymphocyte-depleted subtype that is rich in myeloid lineage cells, though the specific features of this subgroup with respect to myeloid cell composition remains obscure. The purpose of this study was to further dissect myeloid-enriched lung cancers to determine the relative contribution of distinct myeloid lineage cellular populations on lymphocyte infiltration into malignant tumor. Our overarching hypothesis is that identification of the myeloid lineage cellular population most highly associated with lymphocyte depletion would represent a logical secondary therapeutic target with which to combine with anti-PD1/PDL1 antibody therapy.

To accomplish this, we utilized a multiparametric flow data set, Nanostring Immune Profiler gene expression panel, and a potentially novel M-IHC panel to interrogate NSCLC specimens. The M-IHC panel was designed to specifically address potential roles from macrophages, monocytes, M-MDSC, and neutrophils in the mediation of lymphocyte depletion. Of the 4 myeloid cell types assessed, only neutro- 
phils were significantly higher in Myeloid stroma and tumor compared with Active stroma and tumor. As anticipated, $\mathrm{CD}^{+}$cellular content was significantly higher in the stroma and tumor of Active as compared with Myeloid cases. In other words, $\mathrm{CD} 8^{+} \mathrm{T}$ cells were the cellular population most highly associated with immune Active group and neutrophils were the cellular population most highly associated with the Myeloid phenotype. Therefore, we calculated tumor CD8/PMN ratios (using CD8 ${ }^{+} \mathrm{T}$ cell content within malignant tumor and $\mathrm{CD}_{6} 6 \mathrm{~b}^{+}$cellular content within the stroma), in attempts of generating a simple metric capable of phenotyping the immune status of NSCLCs. Indeed, tumor CD8/PMN ratios proved capable of statistically distinguishing immune Active from Myeloid tumors. More importantly, when utilized to interrogate a cohort of ICI-recipient NSCLC patients, tumor CD8/PMN ratios separated CR/PR from both SD and PD patients, and they identified patients displaying the shortest PFS intervals. Furthermore, we were able to validate the ability of CD8/PMN ratios to predict ICI treatment outcomes using gene expression data in an independent cohort. To our knowledge, this is the first report that any myeloid lineage cell population contributes to ICI treatment failures.

In response to the relatively low response rates of anti-PD1 antibody monotherapy, the field had launched over 800 clinical trials by 2016 that combined an anti-PD1/PDL1 antibody with a secondary therapeutic, most commonly a second immune checkpoint (25). Very few of these trials targeted the myeloid lineage, and none of them targeted neutrophils. However, based on the limited efficacy of such trials and data supportive of deleterious roles for myeloid lineage cells, clinical trials addressing these lineages have begun to emerge. Notable examples include combination trials of anti-PD1/PDL1 with CSF1R (NCT02452424), CCR2 (NCT03184870), and CXCR2 (NCT03473925) antagonists. It is within this context that the data reported here take on added value. For example, our data would strongly support the performance of a trial investigating the CXCR2 axis but would not suggest that CCR2 antagonism would be of substantial benefit. At the least, the data presented here should assist investigators with both clinical trial design and interpretation of correlative studies. As a specific example, the finding that all of the patients in the Fred Hutch treatment cohort with high tumor CD8/PMN ratios displayed CR/PRs suggests that neutrophil antagonistic therapies would most likely be of benefit in ICI-refractory patients and would be less likely to benefit patients whose disease recurs after an initial favorable treatment response.

Although we have presented data with respect to the fact that tumor-associated neutrophils are highly associated with lymphocyte depletion and that tumor CD8/PMN ratios predict ICI treatment failures, it should be noted that tumor-associated neutrophils have previously been reported to function deleteriously in NSCLC. Similarly, peripheral blood neutrophil to lymphocyte ratios (NLR) have been used to predict lung cancer mortality and response to treatment. Our group previously reported that tumor neutrophil content was anticorrelated with $\mathrm{CD}^{+}$and $\mathrm{CD} 8^{+} \mathrm{T}$ cell content by flow cytometry (15), a finding that was independently verified by a separate group (26). Additionally, the CIBERSORT group showed that neutrophils were the immune cell population most highly associated with mortality for multiple cancers, including LUAD, using gene expression profiles from > 18,000 patients (27). Multiple studies have found that the peripheral blood NLR predicts patient outcomes in NSCLC, recently summarized in a meta-analysis (28). Most recently, a variant of the NLR that measured Treg content in place of lymphocytes, and PMN-MDSC content (defined as lectin-type oxidized LDL receptor-1 (LOX-1 ${ }^{+}$) PMN located within the peripheral blood mononuclear cell [PBMC] fraction) in place of neutrophils, was used to predict treatment responses to anti-PD1 antibody therapy in NSCLC (29). Certainly, metrics such as the NLR offer useful prognostic information. However, this value can be impacted by numerous cancer-independent variables (e.g., recent infection), and it has not been demonstrated to reflect intratumor immune cell composition, which was directly assessed here. Taken in this context, the current study adds to our understanding of the role of neutrophils in lung cancer with respect to tumor anatomy, relative contribution compared with other myeloid lineage cell types, and therapeutic potential; it also suggests that neutrophils appear to limit the ability of lymphocytes to traffic into malignant tumor. Lastly, there have been well-performed clinical investigations in NSCLC that have demonstrated pro-host functions for tumor-associated neutrophils with respect to lymphocyte function (30). Therefore, the associations drawn here between neutrophils, lymphocyte depletion, and anti-PD1 treatment failures provide solid evidence to target neutrophils in combination with ICI therapy, which has not been uniformly supported by the literature.

To date, most studies addressing ICI treatment outcomes in NSCLC have been focused on metrics capable of identifying the $20 \%$ of patients likely to respond to treatment (8-10). Here, we were able to identify those patients least likely to respond to treatment. Given the inherent nature of the tumor CD8/ 
PMN ratio and its ability to identify these patients, antagonizing PMN activity within CD8/PMN ratiolow tumors may enhance $\mathrm{CD} 8^{+}$cellular content and function, thereby improving treatment outcomes. To directly test this concept, we performed a clinical trial in lung tumor-bearing mice that utilized SX-682, a CXCR1/2 inhibitor, to address the neutrophil lineage. Compared with control mice or those that received anti-PD1 monotherapy, CXCR1/2 inhibitor-treated mice displayed enhanced expression of IFN- $\gamma-$ responsive genes. In other words, CXCR1/2 inhibition effectively converted Myeloid tumors into Active ones. This is with the understanding that immune Active tumors in human NSCLC are still clinically recognizable cancers that continue to grow. Only with the addition of anti-PD1 therapy are reductions in tumor burden achieved, which is what we observed in the combination therapy group (SX-682 plus anti-PD1). Notably, CXCR2 antagonists have previously been reported to enhance the efficacy of anti-PD1 antibodies in various solid tumor models in mice (31-33). This line of experimentation was included here to provide clear evidence that neutrophil antagonism results in the relocation of $\mathrm{CD}^{+} \mathrm{T}$ cells from the tumor periphery into the malignant portions of tumor and restores the IFN- $\gamma$ signature, indicative of favorable immune responses. Ultimately, we believe these results will be utilized to design correlative studies on human NSCLC biospecimens to determine the impact of myeloid-targeting therapies and to drive additional mechanistic interrogation of the interaction between tumor-associated neutrophils and $\mathrm{CD} 8^{+} \mathrm{T}$ cells.

The lack of exome sequencing data represents a limitation in the current study, as we were not able to interrogate the impact of specific mutations and mutational spectral burden on immune phenotypes. A recent report has highlighted an association of mutation in STK11 and anti-PD1 treatment failures (34). Since there are other reports linking STK11 loss with the accumulation of neutrophils in tumors (35), it is possible that STK11 represents a confounder in our data set, and it is an area of active study in our group. Despite the utilization of protein-based flow cytometry and M-IHC assays to enhance gene expression data, our study still possesses limitations in this regard. Since we were limited to 6 markers, there are obvious overlap populations (e.g., $\mathrm{CD} 14^{+} \mathrm{CD} 68^{+}$monocytes) that cannot be fully accounted for. Additionally, we analyzed the macrophage population as a whole using CD68 and, therefore, were unable to assess M1/M2 macrophage subsets here. We attempted to delineate monocytes from M-MDSC using HLA-DR, though the rigor of this approach using M-IHC has not been as clearly validated as it has been for flow cytometry. Lastly, we were unable to distinguish PMN from PMN-MDSC in this study. We attempted to use LOX-1, which has been shown to specifically mark PMN-MDSC in human neutrophils (36), but were unable to validate a LOX-1 antibody in the multispectral format employed here. Optimization of LOX-1 staining for use in M-IHC panels is an ongoing endeavor in our laboratory. Hence, the neutrophil signal measured here using CD66b represents the net sum of PMN and PMN-MDSC. Similarly, just as CD66b is not specific for PMN or PMN-MDSC, CXCR1/2 antagonism is not specific for neutrophils. The use of SX-682 in mice likely impacted not only neutrophil recruitment, but that of monocyte lineages, as well. Use of specific neutrophil-depleting reagents could be utilized to further investigate this issue. Lastly, all mouse models have limitations with respect to the study of immunotherapeutic agents. This is largely related to the limited mutational burden in the genetically engineered models (37). However, we did observe $\mathrm{CD} 8^{+} \mathrm{T}$ cell infiltration into malignant tumor with apparent tumor cell killing (Figure 5E), an important proof-of-concept with respect to this therapeutic strategy.

An additional limitation of our study is that the treatment cohort employed was relatively small. Accordingly, the final number of patients evaluable for treatment outcomes was $n=28$. We have attempted to overcome this limitation by using an independent validation cohort, though this required the utilization of gene expression data as opposed to IHC. We undertook this study to further interrogate the role of individual myeloid lineage cell types within the lung TME and to determine their impact on immune phenotypes and ICI treatment outcomes. As such, the signatures and metrics employed here were utilized for their ability to identify the myeloid lineage cell most likely responsible for prohibiting lymphocyte access to malignant tumor and not for their ability to predict treatment outcomes, per se. Therefore, the ability of the M-IHC-based tumor $\mathrm{CD} 8 / \mathrm{PMN}$ ratio to predict ICI treatment outcome should be validated in an independent cohort.

This is the seminal report that neutrophil lineage cells highlight the Myeloid phenotype and contribute to anti-PD1/PDL1 treatment failures in NSCLC patients. When combined with the results of the mouse clinical trial, the human tissue data strongly support a formal clinical investigation of the ability of neutrophil inhibition to enhance the efficacy of ICI therapy in NSCLC patients. Currently, most of the potentially efficacious neutrophil antagonizing therapeutics have either addressed just one of many CXCR1/2 ligands (e.g., CXCL-8) or are CXCR2 specific. Given its ability to address both CXCR1 and CXCR2 (and therefore all of the relevant CXCLs), SX-682 may prove more efficacious in this regard than other agents in this class. 


\section{Methods}

Study design. The current study utilizes 3 distinct patient cohorts and 1 mouse model of lung cancer. The first patient cohort used was a previously described cohort of $n=73$ NSCLC patients undergoing lung cancer resection for curative intent (15). This cohort has been termed the resection cohort. Here, we analyzed the previously reported flow cytometric data set using unbiased analytical approaches. Additionally, we obtained formalin-fixed paraffin-embedded (FFPE) blocks from this cohort for the purposes of potentially new gene expression studies and potentially new M-IHC studies. These assays are described in detail below. This research was performed on consented subjects at the Fred Hutchinson Cancer Research Center (FHCRC)/University of Washington Hospital/Northwest BioTrust, under an active IRB protocol.

The second patient cohort consisted of $n=28$ NSCLC patients treated at the Seattle Cancer Care Alliance (SCCA) with either anti-PD1 or anti-PDL1 antibody therapy as an approved or investigational therapy between 2012 and 2017 and for which pretreatment FFPE tissue was available (Supplemental Table 1). This cohort has been termed the Fred Hutch Treatment cohort. Slides were subjected to staining and analysis as described below. RECIST V1.1 status was determined for each patient utilizing available imaging and as previously described (22). Additionally, we calculated the PFS interval for each patient using the date of anti-PD1/anti-PDL1 treatment initiation and date at which recurrence or progression was identified on imaging. Patients were censored if they had not been diagnosed with disease progression at the time of analysis. All patients consented to having their clinical data archived or fresh biopsy specimens accessed. These studies were performed under an active IRB protocol.

The third cohort consisted of $n=52$ NSCLC patients treated with second-line anti-PD1/PDL1 therapy at the University of Pennsylvania. This cohort is referred to as the UPenn Treatment Cohort. Treatment response category was assigned according to RECIST V1.1 criteria and rendered $n=9 \mathrm{CR} / \mathrm{PR}, n=16$ $\mathrm{SD}$, and $n=27 \mathrm{PD}$. FFPE slides were available for each patient and were subjected to the HTG EdgeSeq Precision Immuno-Oncology panel (VERI/O Laboratory Services), a gene expression platform capable of measuring 1,410 target genes. CD8 scores were generated using normalized gene expression data. $C D 8 A$ and GZMB and PMN scores were generated using S100A8, S100A9, and KRT23. CD8/PMN ratios were generated using these 2 scores. These studies were performed under an active IRB protocol.

Flow cytometry. The performance of flow cytometry, including specimen acquisition and processing, antibodies utilized, and gating strategy, have all been previously described in detail $(15,38)$. Briefly, single cell suspensions were generated from lung cancer tissue specimens and nonadjacent tumor-free lung tissue and subjected to multiple flow cytometric panels that encompassed 27 antibodies capable of identifying 51 immune cell populations and subpopulations. The numerical data for these 51 cellular populations were utilized for analytical purposes here.

Nanostring gene expression profiles. RNA was extracted from $50-\mu \mathrm{m}$ "curls" cut from FFPE blocks of resection cohort patients using standard Qiagen RNeasy FFPE kits, as per protocol. RNA was subjected to the Nanostring Immune Profiling panel. The specimens were run on an nCounter as per manufacturer's instructions. Normalization and batch correction were carried out to apply a sample-specific correction factor to all the target probes per manufacturer's recommendations (Positive Control Normalization using synthetic positive control targets and CodeSet Content Normalization, using housekeeping genes). For the pairwise expression comparisons, the scales represent $\log _{2}$ (expression), where the units of expression are arbitrary because they represent barcode counts after rescaling for normalization and batch effect correction. For the Nanostring data heatmaps, the above values were turned into Z-scores and truncated to within \pm 2 to reduce the effects of outlier values.

Processing of TCGA data. mRNA abundance of genes measured in reads per kilobase of transcript, per million mapped reads (RPKM) units from 57 tumor-normal LUAD pairs were accessed from the TCGA (39). Data were $\log$ transformed, and then each cell marker gene was normalized across samples to the sample-specific CD45 values by linear regression separately for cancer and control samples: $\log (G e n e)=b 0+b 1 \times \log (C D 45)$. The residuals were used to represent the normalized values, which center each gene as having mean zero. Data were scaled so that each marker has mean zero and variance 1. All FACS data were expressed as percent of CD45 and log transformed to be comparable with the gene expression assay. The expression data and the FACS data, by this transformation, are now on a scale relative to CD45 as gene expression data sets.

Gene expression data of MMP8, CD8A, and CD14 were used for TAN, CD8, and monocyte populations, respectively. A total of 319 tumor samples were used to fit the linear regression model PMN/ CD8 $\left(P=6.18 \times 10^{-5}\right)$, and 355 tumor samples were used to fit the linear regression model CD14/CD8 $\left(P<2 \times 10^{-16}\right)$. 
M-IHC. All M-IHC staining and MSI was performed using PerkinElmer's Opal fIHC reagents and the Vectra-3 Multispectral Imaging System (PerkinElmer). Slides obtained from FFPE blocks were used for these studies that employed 2 separate panels for human tissues and 1 panel for mouse tissue. Panel 1 was utilized to study the resection cohort, Panel 2 was used to assess the Treatment cohort, and Panel 3 was used to study mouse tumors. The staining methodology and analytical strategies were the same for all panels and are described below. The antibodies utilized are listed in Supplemental Table 2.

FFPE tumor tissue slides sectioned at $4 \mu \mathrm{m}$ were baked for 1 hour at $60^{\circ} \mathrm{C}$; they were then dewaxed and stained on a Leica BOND Rx stainer (Leica). Leica Bond reagents were used for dewaxing (Dewax Solution), antigen retrieval, antibody stripping (Epitope Retrieval Solution 2), and rinsing after each step (Bond Wash Solution). A high-stringency wash was performed after the secondary and tertiary applications using high-salt TBST solution (0.05 M Tris, 0.3 M NaCl, and 0.1\% Tween-20, pH 7.2-7.6). OPAL Polymer HRP Mouse plus Rabbit (PerkinElmer) was used for all secondary applications for human tissues, and ImmPress HRP anti-rat IgG (Vector Lab, MP-7451) polymers were used for mouse tumors. Antigen retrieval and antibody stripping steps were performed at $100^{\circ} \mathrm{C}$ with all other steps at room temperature. Endogenous peroxidase was blocked with $3 \% \mathrm{H}_{2} \mathrm{O}_{2}$ for 8 minutes, followed by protein blocking with TCT buffer (0.05 M Tris, $0.15 \mathrm{M} \mathrm{NaCl}, 0.25 \%$ Casein, 0.1\% Tween-20, pH $7.6 \pm 0.1)$ for 30 minutes. The first primary antibody (Position 1) was applied for 60 minutes, followed by the secondary antibody application for 10 minutes and the application of the tertiary TSA-amplification reagent (PerkinElmer OPAL fluor) for 10 minutes. The primary and secondary antibodies were stripped with retrieval solution for 20 minutes before repeating the process with the second primary antibody (Position 2), starting with a new application of $3 \% \mathrm{H}_{2} \mathrm{O}_{2}$. The process was repeated until all 6 positions were completed; there was not a stripping step after Position 6. The sequence of antibody staining was tested and optimized for both panels in a series of experiments depicted in Supplemental Figure 2 and Supplemental Table 3.

Slides were removed from the stainer and stained with Spectral DAPI (Perkin Elmer) for 5 minutes, rinsed for 5 minutes, and cover-slipped with Prolong Gold Antifade reagent (Invitrogen). Stained slides were cured for 24 hours at room temperature, and MSI of representative tumor tissue areas from each slide were acquired on either the PerkinElmer Vectra 3.0 Automated Imaging System for 6-plex and 4-plex images or Aperio FL (Leica) for 3-plex images. Vectra images were spectrally unmixed using PerkinElmer InForm software and exported as multiimage TIFFs for analysis in HALO software (Indica Labs). Aperio FL images were imported directly into the HALO software for analysis.

Based on tissue section size, multiple stamps (at least 20 ROI per slide) were captured in a geographically unbiased and random fashion to direct deeper scanning at $\times 20$ for acquisition of MSI. ROI fields were 1,338 $\mu \mathrm{m}$ by $1,000 \mu \mathrm{m}$ in size. Vascular structures were excluded from the analysis. We ensured that key tumor structures were captured from each slide, including areas of tumor/stroma interface, central tumor, necrotic tumor, LA, and adjacent lung tissue. These histological features were identified using characteristic tissue and staining attributes. Tumor cells were identified by being tumor marker (cytokeratin) positive and by their unique cell morphology. Based on tumor histology and tissue structure formed by densely interconnected tumor cell clusters, several tissue compartments were determined. Area occupied by $\mathrm{CK}^{+}$tumor cells was defined as malignant tumor, and areas devoid of tumor cells but possessing targeted immune cells and other cells types, along with the presence of vascular structures, were defined as stroma. Necrotic tumor areas were defined by tissue destruction, apparently dead tumor cell morphology, and densely accumulated CD66 $\mathrm{b}^{+}$cell clusters, which were used as guides to draw the edge of the necrotic region. LA were defined by characteristic cell clustering shapes (germinal center like) formed by densely packed small and round DAPI ${ }^{+}$cells mixed with $\mathrm{CD} 8^{+}$cells. Lastly, adjacent lung tissue was marked by the presence of alveolar structures.

Cells were detected based on nuclear recognition (DAPI stain), and the fluorescence intensity of the cytoplasmic areas of each cell was measured. A mean intensity threshold above background was used to determine positivity for each fluorochrome within the cytoplasm, thereby defining cells as either positive or negative for each marker. The positive cell data was then used to define colocalized populations and to perform spatial analysis. Final HALO analysis results were exported, and data tabulation was done with a custom-written Java script. Each cell type-specific signal was quantified as the number of positively stained cells for the total image, stroma, tumor, necrotic tumor, LA, and adjacent lung tissue (expressed as cells/ $\mathrm{mm}^{2}$ tissue). The analytical process is depicted in detail in Supplemental Figure 4.

Data analysis. The heatmap in Figure 1A was generated using the Z-scores of percent-live flow cytometry measurements, Z-score values outside \pm 2 were truncated to limit the effects of outlier values. All pairwise 
expression comparisons were performed using a 2-tailed $t$ test. The heatmap in Figure $3 \mathrm{C}$ was generated as above using cell counts $/ \mu \mathrm{m}^{2}$ of tumor tissue from M-IHC-stained slides. The Nanostring gene expression heatmap in Figure 1B was generated using the Z-scores of $\log _{2}$ expression values of Nanostring probe counts after normalization and batch correction per manufacturer's specification. Z-scores outside \pm 2 were truncated to limit the effects of outlier values. The data in Supplemental Figure 1A were generated using a classical multidimensional scaling (MDS) sample similarity pot. Distances were calculated from Pearson correlation values using the "rcorr" function of the "hmisc" R package (https://cran.r-project.org/web/packages/Hmisc).

Mice. All mouse experiments reported here utilized age- and sex-matched mice and were performed at the Fred Hutchinson Cancer Research Center under protocols approved by the IACUC. Pten fl/fl and Lkb1 $1^{f / f l}$ mice were both acquired from the Jackson Laboratory on a pure C57BL/ 6 background. PL mice were generated by simple cross-breeding. To initiate tumorigenesis, the mice were treated with a single intratracheal dose of AdCre at a titer of $5 \times 10^{7} \mathrm{pfu}$ (University of Iowa Viral Vector Core, Iowa City, Iowa, USA), as previously described (40). After allowing 30 weeks for tumor development, the mice underwent MRI using a Bruker 1T MRI machine (Bruker Instruments). The index tumors were measured using OsiriX DIACOM viewer. The tumor volume was calculated by measuring the sum of stacked disk area from the MRI scans of each tumor. At this time, each mouse was assigned to 1 of 4 treatment groups: (a) anti-PD1 antibody (BioXcel, clone RMP1-14, 10 mg/kg 3×/week), (b) SX-682 (CXCR1/2 inhibitor, Syntrix Biosystems) impregnated food (500 mg/kg/day), (c) anti-PD1 plus SX-682, or (d) isotype control. Each mouse was reimaged using MRI following 4 weeks of treatment. The MRI scans were utilized to measure differences in tumor burden as a function of treatment group.

Tumor specimens were either resected from mice and frozen or fixed in formalin, to allow for further studies. RNA was isolated from frozen tumor specimens using TRIzol (Invitrogen) and subsequently purified with a standard kit (RNeasy Mini Kit, QIAGEN). The RNA ( $1 \mu \mathrm{g})$ was reverse transcribed using SuperScript II Reverse Transcriptase (Invitrogen). The expression of the following genes was assessed using TaqMan primer/probe sets (Applied Biosystems): Ifn $\gamma$, Cxcl9, Cxcl10, Ilr2, Gzmb, and Stat1. qPCR analysis was performed using a StepOnePlus Real-Time PCR System (Invitrogen). All reactions were performed in triplicate. The $\Delta \mathrm{Ct}$ method was employed utilizing Gapdh as the endogenous housekeeping gene. FFPE tumor sections were subjected to M-IHC staining and analysis as described above.

Statistics. All data were expressed as the mean value \pm SEM, unless otherwise indicated. In some instances, not all $n=73$ cases in the resection cohort were evaluable for a given metric (e.g., poor RNA quality). Therefore, the actual $N$ for each analysis is listed throughout the study. Multiple comparisons were assessed using 1-way ANOVA with Tukey post hoc test. Paired cell analysis depicted in Figure 4, C and D, was analyzed using a 2-way ANOVA. Head-to-head comparisons were assessed using a 2-tailed Student's $t$ test. Differences in survival were depicted using Kaplan-Meier life survival curves, and statistical significance was assessed using the Log-rank test. $P<0.05$ was considered significant.

Study approval. All human studies were reviewed and approved by the Fred Hutchinson Cancer Research Center IRB. All animal experiments were reviewed and approved by the Fred Hutchinson Cancer Research Center IACUC.

\section{Author contributions}

JK designed, performed, analyzed, and interpreted experiments. XZ generated, performed, and analyzed all M-IHC assays and data. HZ performed and analyzed all mouse experiments and data. GHHY and JMF performed experiments and analyzed data. TJF and RHP assisted conceptualization. MS and CB acquired IRB approval and established clinical cohorts. DYM and JAZ provided SX-682 and assisted with data interpretation. JMF, GM, and A. Mashadi-Hossein analyzed gene expression data. HB analyzed all of the data. MWR performed statistical analyses. JCT and SMA generated all data associated with the UPenn Treatment Cohort. JK and A.M. Houghton designed the project. A.M. Houghton crafted the figures and wrote the manuscript.

\section{Acknowledgments}

We thank the Fred Hutch Flow Cytometry and Experimental Histopathology Shared Resources. We also thank Kim Melton for her assistance with development of the M-IHC panels, Kimberly S. Smythe for her assistance in image acquisition and HALO analysis, and the NorthwestBioTrust for specimen procurement. 
This work was supported by grants from the NIH (R01CA223191 and P50CA228944 to AMH) and the Seattle Translational Tumor Research (STTR) program (to AMH). JK was supported by European Commission grant EU-FP7-PEOPLE-2012-IOF 331255

Address correspondence to: A. McGarry Houghton, Clinical Research Division, Fred Hutchinson Cancer Research Center, 1100 Fairview Avenue North, D4-100, Seattle, Washington 98109, USA. Phone: 206.667.3175; Email: houghton@fredhutch.org.

1. Siegel R, Ma J, Zou Z, Jemal A. Cancer statistics, 2014. CA Cancer J Clin. 2014;64(1):9-29.

2. Topalian SL, et al. Safety, activity, and immune correlates of anti-PD-1 antibody in cancer. N Engl J Med. 2012;366(26):2443-2454.

3. Brahmer J, et al. Nivolumab versus Docetaxel in Advanced Squamous-Cell Non-Small-Cell Lung Cancer. N Engl J Med. 2015;373(2):123-135.

4. Borghaei H, et al. Nivolumab versus Docetaxel in Advanced Nonsquamous Non-Small-Cell Lung Cancer. N Engl J Med. 2015;373(17):1627-1639.

5. Reck M, et al. Pembrolizumab versus Chemotherapy for PD-L1-Positive Non-Small-Cell Lung Cancer. NEngl J Med. 2016;375(19):1823-1833.

6. Gandhi L, et al. Pembrolizumab plus Chemotherapy in Metastatic Non-Small-Cell Lung Cancer. N Engl J Med. 2018;378(22):2078-2092.

7. Brahmer JR, et al. Safety and activity of anti-PD-L1 antibody in patients with advanced cancer. $N$ Engl J Med. 2012;366(26):2455-2465.

8. Rizvi NA, et al. Cancer immunology. Mutational landscape determines sensitivity to PD-1 blockade in non-small cell lung cancer. Science. 2015;348(6230):124-128.

9. Tumeh PC, et al. PD-1 blockade induces responses by inhibiting adaptive immune resistance. Nature. 2014;515(7528):568-571

10. Ayers M, et al. IFN- $\gamma$-related mRNA profile predicts clinical response to PD-1 blockade. J Clin Invest. 2017;127(8):2930-2940.

11. Haanen JBAG. Converting Cold into Hot Tumors by Combining Immunotherapies. Cell. 2017;170(6):1055-1056.

12. Binnewies M, et al. Understanding the tumor immune microenvironment (TIME) for effective therapy. Nat Med. 2018;24(5):541-550

13. Thorsson V, et al. The Immune Landscape of Cancer. Immunity. 2018;48(4):812-830.e14.

14. Jiang P, et al. Signatures of T cell dysfunction and exclusion predict cancer immunotherapy response. Nat Med. 2018;24(10):1550-1558.

15. Kargl J, et al. Neutrophils dominate the immune cell composition in non-small cell lung cancer. Nat Commun. 2017;8:14381

16. Takeda A, et al. Cutting edge: role of IL-27/WSX-1 signaling for induction of T-bet through activation of STAT1 during initial Th1 commitment. J Immunol. 2003;170(10):4886-4890.

17. Chakraborty S, Kaur S, Guha S, Batra SK. The multifaceted roles of neutrophil gelatinase associated lipocalin (NGAL) in inflammation and cancer. Biochim Biophys Acta. 2012;1826(1):129-169.

18. Condamine T, et al. ER stress regulates myeloid-derived suppressor cell fate through TRAIL-R-mediated apoptosis. J Clin Invest. 2014;124(6):2626-2639.

19. Galon J, et al. Type, density, and location of immune cells within human colorectal tumors predict clinical outcome. Science. 2006;313(5795):1960-1964.

20. Galon J, et al. Cancer classification using the Immunoscore: a worldwide task force. J Transl Med. 2012;10:205.

21. Bronte V, et al. Recommendations for myeloid-derived suppressor cell nomenclature and characterization standards. Nature communications. 2016;7:12150.

22. Eisenhauer EA, et al. New response evaluation criteria in solid tumours: revised RECIST guideline (version 1.1). Eur J Cancer. 2009;45(2):228-247.

23. Lu X, et al. Effective combinatorial immunotherapy for castration-resistant prostate cancer. Nature. 2017;543(7647):728-732

24. Xu C, et al. Loss of Lkb1 and Pten leads to lung squamous cell carcinoma with elevated PD-L1 expression. Cancer Cell. 2014;25(5):590-604.

25. Brawley L. With 20 Agents, 803 Trials, and 166,736 Patient Slots, Is Pharma Investing Too Heavily in PD-1 Drug Development? Cancer Lett. 2016;42(37):2-18.

26. Lizotte PH, et al. Multiparametric profiling of non-small-cell lung cancers reveals distinct immunophenotypes. JCI Insight. 2016;1(14):e89014.

27. Gentles AJ, et al. The prognostic landscape of genes and infiltrating immune cells across human cancers. Nat Med. 2015;21(8):938-945.

28. Yu Y, Qian L, Cui J. Value of neutrophil-to-lymphocyte ratio for predicting lung cancer prognosis: A meta-analysis of 7,219 patients. Mol Clin Oncol. 2017;7(3):498-506

29. Kim HR, et al. The Ratio of Peripheral Regulatory T Cells to Lox-1 $1^{+}$Polymorphonuclear Myeloid-derived Suppressor Cells Predicts the Early Response to Anti-PD-1 Therapy in Patients with Non-Small Cell Lung Cancer. Am J Respir Crit Care Med. 2019;199(2):243-246.

30. Eruslanov EB, et al. Tumor-associated neutrophils stimulate T cell responses in early-stage human lung cancer. J Clin Invest. 2014;124(12):5466-5480.

31. Steele CW, et al. CXCR2 Inhibition Profoundly Suppresses Metastases and Augments Immunotherapy in Pancreatic Ductal Adenocarcinoma. Cancer Cell. 2016;29(6):832-845.

32. Liao W, et al. KRAS-IRF2 Axis Drives Immune Suppression and Immune Therapy Resistance in Colorectal Cancer. Cancer Cell. 2019;35(4):559-572.e7.

33. Sun L, et al. Inhibiting myeloid-derived suppressor cell trafficking enhances $\mathrm{T}$ cell immunotherapy. JCI Insight. 
2019;4(7):126853

34. Skoulidis F, et al. STK11/LKB1 Mutations and PD-1 Inhibitor Resistance in KRAS-Mutant Lung Adenocarcinoma. Cancer Discov. 2018;8(7):822-835.

35. Mollaoglu G, et al. The Lineage-Defining Transcription Factors SOX2 and NKX2-1 Determine Lung Cancer Cell Fate and Shape the Tumor Immune Microenvironment. Immunity. 2018;49(4):764-779.e9.

36. Condamine T, et al. Lectin-type oxidized LDL receptor-1 distinguishes population of human polymorphonuclear myeloid-derived suppressor cells in cancer patients. Sci Immunol. 2016;1(2):aaf8943.

37. Westcott PM, et al. The mutational landscapes of genetic and chemical models of Kras-driven lung cancer. Nature. 2015;517(7535):489-492.

38. Mark NM, et al. Chronic Obstructive Pulmonary Disease Alters Immune Cell Composition and Immune Checkpoint Inhibitor Efficacy in Non-Small Cell Lung Cancer. Am J Respir Crit Care Med. 2018;197(3):325-336.

39. Ellrott K, Omberg L. Synapse TCGA Pancancer project. http://www.synapse.org/\#!Synapse:syn300013. Updated August 11, 2019. Accessed November 20, 2019.

40. Busch SE, Hanke ML, Kargl J, Metz HE, MacPherson D, Houghton AM. Lung Cancer Subtypes Generate Unique Immune Responses. J Immunol. 2016;197(11):4493-4503. 